\title{
Smart Hydrogel Micromechanical Resonators with Ultrasound Readout for Biomedical Sensing
}

\author{
Navid Farhoudi ${ }^{1}$, Hsuan-Yu Leu ${ }^{2}$, Lars B. Laurentius ${ }^{1}$, Jules J. Magda ${ }^{2}$, Florian Solzbacher ${ }^{1,3,4}$, Christopher F. Reiche ${ }^{1}$ \\ ${ }^{1}$ Department of Electrical and Computer Engineering, University of Utah, Salt Lake City, 84112, USA \\ ${ }^{2}$ Department of Chemical Engineering, University of Utah, Salt Lake City, 84112, USA \\ ${ }^{3}$ Department of Materials Science \& Engineering, University of Utah, Salt Lake City, UT 84112, USA \\ ${ }^{4}$ Department of Bioengineering, University of Utah, Salt Lake City, UT 84112, USA \\ Content: \\ - Preparation of pre-gel solutions \\ - Preparation of test solutions \\ - Preparation of phosphate-buffered saline solutions \\ - The effect of bubbles on the offset level of the in vitro results \\ - Additional in vitro salt concentration response
}

Preparation of phosphate-buffered saline solutions. Phosphate-buffered saline (PBS) solutions used in all experiments were prepared by dissolving $8.000 \mathrm{~g}$ of $\mathrm{NaCl}$ (Sigma-Aldrich), $0.200 \mathrm{~g}$ of KCl (Sigma-Aldrich), 1.440 $\mathrm{g}$ of $\mathrm{Na}_{2} \mathrm{HPO}_{4}$ (Sigma-Aldrich), and $0.240 \mathrm{~g}$ of $\mathrm{KH}_{2} \mathrm{PO}_{4}$ (Sigma-Aldrich) in $\sim 900 \mathrm{~mL}$ of DI water, after which the $\mathrm{pH}$ was adjusted to 7.40 at $20{ }^{\circ} \mathrm{C}$ by adding a proper amount of $1 \mathrm{M} \mathrm{HCl}$ or $\mathrm{NaOH}$ as needed. The solution was then transferred to a volumetric flask, and the total volume adjusted to $1 \mathrm{~L}$ by adding DI water. This formulation (containing $10 \mathrm{mM}$ sodium phosphate, $1.8 \mathrm{mM}$ potassium phosphate, $137 \mathrm{mM} \mathrm{NaCl}$, and $2.7 \mathrm{mM} \mathrm{KCl}$ ) corresponds to a $1 \times \mathrm{PBS}$ solution. Lower concentrations of PBS were prepared by diluting the $1 \times \mathrm{PBS}$ with DI water. For example, $1 / 4 \times \mathrm{PBS}$ is the result of a 1:3 dilution of $1 \times \mathrm{PBS}$ with DI water. DI water was used with a resistivity of $\sim 16 \mathrm{M} \Omega-\mathrm{cm}$.

Preparation of glucose solutions. The different concentrations of glucose in PBS buffer were made by diluting a concentrated stock solution of glucose $(500 \mathrm{mM})$ in $1 \times \mathrm{PBS}$ by appropriate amounts of $1 \times \mathrm{PBS}$. The stock solution was made by dissolving $18.106 \mathrm{~g}$ of dextrose (Sigma-Aldrich) in $180 \mathrm{~mL}$ of $1 \times \mathrm{PBS}$ solution and then increasing the volume to $200 \mathrm{~mL}$ by adding $1 \times$ PBS.

Preparation of pre-gel solutions. The polyampholytic smart hydrogels used in this study are sensitive to glucose as well as $\mathrm{pH}$ and ionic strength. The pre-gel monomer solution was prepared as described by Leu et al. ${ }^{1}$ with slight modifications. In short, we first used DI water to prepare a $1 \mathrm{mM}$ buffer solution of 4-(2hydroxyethyl)piperazine-1-ethanesulfonic acid (HEPES, Sigma-Aldrich). The $\mathrm{pH}$ of the buffer was adjusted to 8.0 at $20{ }^{\circ} \mathrm{C}$ by adding a proper amount of $1 \mathrm{M} \mathrm{HCl}$ or $\mathrm{NaOH}$ as needed. An appropriate amount of acrylamide (Fisher Scientific) was added to this $1 \mathrm{mM}$ HEPES buffer to obtain a $30 \% \mathrm{w} / \mathrm{w}$ monomer solution. Next $19.1 \mathrm{mg}$ of the anionic monomer 3-acrylamidophenylboronic acid (Frontier Scientific, Inc) was dissolved in $87 \mu \mathrm{L}$ of dimethyl sulfoxide (Sigma-Aldrich), then mixed with $237 \mu \mathrm{L}$ of the $30 \% \mathrm{w} / \mathrm{w}$ solution of acrylamide in $1 \mathrm{mM}$ HEPES buffer, $193 \mu \mathrm{L}$ of $2 \% \mathrm{w} / \mathrm{w}$ solution of the crosslinker N,N'-methylenebisacrylamide (Sigma-Aldrich), $309 \mu \mathrm{L}$ of 1 $\mathrm{mM}$ HEPES buffer, and $20.48 \mu \mathrm{L}$ of the cationic monomer N-[3-(dimethylamino)propyl]methacrylamide (SigmaAldrich), in order. In addition, to this monomer solution was added $25.8 \mu \mathrm{L}$ of $4 \% \mathrm{w} / \mathrm{w}$ solution of photoinitiator lithium phenyl-2,4,6-trimethylbenzoylphosphinate (Sigma-Aldrich) in 1 mM HEPES. 
The effect of bubbles on the offset level of the in vitro results. The MGV for the hydrogel pillars structure in the main text at $9 \mathrm{MHz}$ frequency has sudden offset changes in the time of solution exchanges. The offset change in the signal could be attributed to possible bubbles introduced to the solution due to the manual process of the solution exchange. The presence of bubbles could create high contrast regions in the ultrasound image and affect the overall grayscale value of the pixels corresponding to the spatial location of the bubble. Although these bubbles can change the overall offset of the MGV in their surroundings, they do not block the signals from the hydrogel structures due to significantly bigger area of the hydrogel compared to the size of the bubbles, as well as, due to the fact that they do not change over the measurement duration and only happen in solution exchanges. Figure S1 shows two exemplary B-Mode ultrasound images of the hydrogel pillar structure from before and immediately after a solution exchange. These images are chosen where the solution exchange has created a change in the offset of the MGV in $9 \mathrm{MHz}$ imaging frequency.

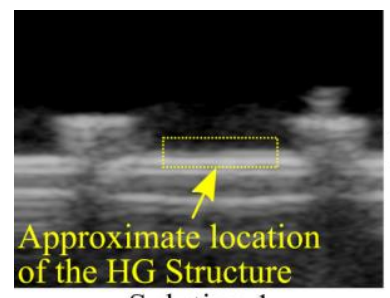

Solution 1

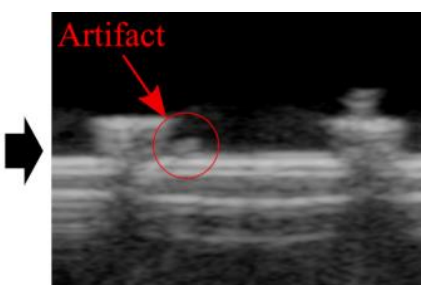

Solution 2

Figure S1: The appearance of an artifact in the vicinity of the hydrogel resonator structure (hydrogel sheet with hydrogel pillars) which causes a change in the offset level at $9 \mathrm{MHz}$ frequency.

It is worth noting that these bubbles affect higher imaging frequencies more, and it is less disruptive to lower frequencies because higher frequencies have higher imaging resolution and are therefore more prone to show the effect of smaller bubbles in the image.

Additional in vitro salt concentration response. The hydrogel pillar-backplane structure that was used for the measurements in Figure 5 and 6 in the main text was also used to measure the changes in the salt concentrations (see supplementary Figure S2) in the same method described for the results presented in Figure 4 of the main publication. The $4 \mathrm{MHz}$ response of this HG structure is comparable to the results shown in Figure 4 of in the main text. While the 6 and $9 \mathrm{MHz}$ responses also follow this trend, they are strongly affected by experimental noise, possibly due to the presence of air bubbles in the PBS solution.

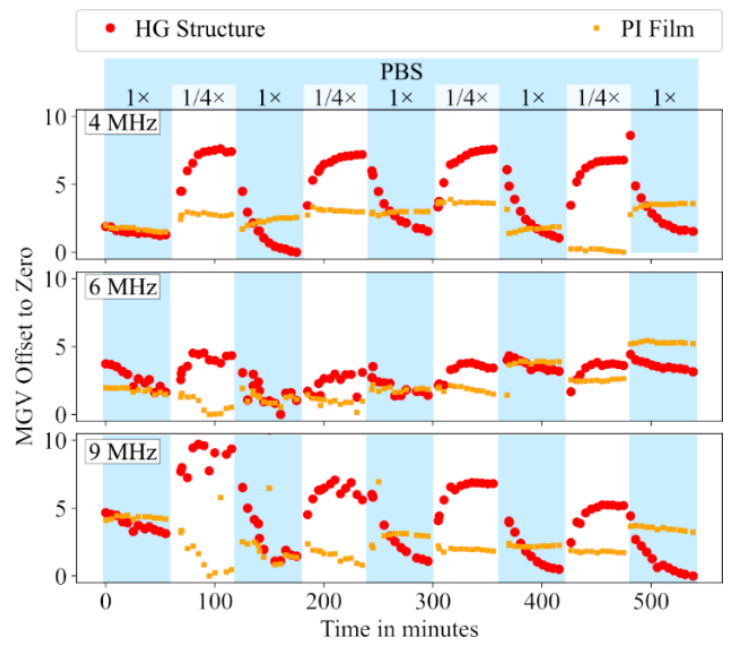

Figure S2: The response of a combined microresonator structure (hydrogel pillars-backplane, same as used for data in figure 5 and 6 in the main publication) and a polyimide (PI) control sample for transitions between two different concentrations of PBS as recorded at 4 $\mathrm{MHz}, 6 \mathrm{MHz}$, and $9 \mathrm{MHz}$, respectively 


\section{References:}

(1) Leu, H.-Y.; Farhoudi, N.; Reiche, C.; Körner, J.; Mohanty, S.; Solzbacher, F.; Magda, J. Low-Cost Microfluidic Sensors with Smart Hydrogel Patterned Arrays Using Electronic Resistive Channel Sensing for Readout. Gels 2018, 4 (4), 84. https://doi.org/10.3390/gels4040084. 